\title{
The negative effect of ANGPTL8 on HDL-mediated cholesterol efflux capacity
}

\author{
Mengdie Luo ${ }^{1}$, Ziyu Zhang ${ }^{1}$, Yani Peng ${ }^{2}$, Shuai Wang ${ }^{1}$ and Daoquan Peng ${ }^{1 *}$
}

\begin{abstract}
Background: It is well known that angiopoietin-like protein 8 (ANGPTL8) exerts its effects on lipid metabolism through the inhibition of lipoprotein lipase and subsequent elevation of plasma triglyceride. However, it is not clear whether ANGPTL8 could affect lipid metabolism via other pathways. The study was aimed to investigate the effects of ANGPTL8 on the function of high-density lipoprotein (HDL), which plays a protective role in atherosclerosis progression.

Methods: Two hundred and ten subjects were recruited. Plasma ANGPTL8 was measured by enzyme-linked immunosorbent assays. Cholesterol efflux capacity was chosen as the biomarker of HDL function and measured via $\mathrm{H}^{3}$-cholesterol loading THP-1 cell models.

Results: ANGPTL8 exhibited no significant difference between CAD group and nonCAD group, but ANGPTL8 in DM group was significantly higher than that in the nonDM group [568.3 (406.2-836.8) vs 458.2 (356.8-755.6), P=0.023]. Compared to controls, subjects in CAD group and DM group exhibited significantly lower cholesterol efflux capacity [CAD: $14.58 \pm 2.06$ vs $12.51 \pm 2.83 \%, P<0.0001 ; \mathrm{DM}: 13.62 \pm 2.57$ vs $12.34 \pm 3.16 \%, P=0.0099]$. ANGPTL8 was inversely correlated with cholesterol efflux capacity $(r=-0.188, P<0.01)$. Regression analysis revealed that plasma ANGPTL8 was an independent contributor to cholesterol efflux capacity (standardized $\beta=-0.143, P=0.023$ ).
\end{abstract}

Conclusion: ANGPTL8 presents a negative effect on HDL-mediated cholesterol efflux capacity.

Keywords: ANGPTL8, Cholesterol efflux capacity, Coronary artery disease, Diabetes

\section{Background}

Many epidemiological studies revealed that high-density lipoprotein cholesterol (HDL-C) level was inversely correlated with CAD incidence and presented HDL-C as a robust biomarker of cardiovascular risk [1,2]. However, clinical trials [3, 4] and Mendelian randomization studies [5] failed to prove the causative association of HDL-C and clinical benefits. Due to the disappointing outcomes of raising HDL-C and the heterogeneity of components across HDL particles, HDL functionality emerged as a more intriguing target. Among multiple atheroprotective effects of HDL, its participation in reverse cholesterol transport (RCT) was demonstrated to be the most

\footnotetext{
*Correspondence: pengdq@csu.edu.cn

${ }^{1}$ Department of Cardiovascular Medicine, The Second Xiangya Hospital, Central South University, No.139, Middle Renmin Road, Changsha 410011, Hunan, China

Full list of author information is available at the end of the article
}

important [6]. Cholesterol efflux from peripheral tissue and cells, the initial step of RCT, presented a strongly inverse association with carotid intima-media thickness and CAD likelihood independent of HDL-C level [7]. Our previous study in patients with chronic kidney diseases also confirmed the reverse correlation between cholesterol efflux and carotid intima-media thickness [8]. A prospective cohort study revealed that HDL-mediated cholesterol efflux capacity, instead of HDL-C level, was an independent predictor of CAD incidence [9]. Therefore, it may help the development of therapeutic interventions to unravel the molecular determinants of HDL-mediated cholesterol efflux capacity and improve HDL function [10].

The angiopoietin-like proteins (ANGPTL1-8) are secreted glycoproteins sharing common structures but exerting distinct physiological effects [11]. ANGPTL8, also referred to as betatrophin, lipasin, refeeding-induced 
in fat and liver (RIFL) and hepatocellular carcinomaassociated protein (TD26), was found to be a novel player in lipid metabolism [12]. ANGPTL8, together with ANGPTL3 and ANGPTL4, could regulate triglyceride metabolism by inhibiting the activity of lipoprotein lipase (LPL) [13, 14], the rate-limiting enzyme for triglyceride hydrolysis and plasma triglyceride clearance [15]. Genome-wide association studies (GWAS) in American Indians and Mexican Americans identified that ANGPTL8 variant was associated with HDL-C level [16]. A cross-sectional study targeted at Japanese subjects also found that increased ANGPTL8 concentration was inversely correlated with circulating HDL-C [17]. Another study compared the change in serum ANGPTL8 concentration after bariatric surgery and found that the change in ANGPTL8 concentration was positively correlated with change in HDL-C concentration [18]. Although many studies have studied the relationship between ANGPTL8 and HDL-C, however, it is still unclear whether ANGPTL8 could affect HDL function. As mentioned above, HDL-mediated cholesterol efflux capacity is a significant surrogate of HDL function. Therefore, in the present study, we conducted an observational study to examine the relationship between ANGPTL8 and HDLmediated cholesterol efflux capacity.

\section{Methods}

\section{Subjects}

We recruited $120 \mathrm{CAD}$ subjects and 90 nonCAD subjects from the Department of Cardiovascular Medicine of Second Xiangya Hospital, Central South University. In this study, CAD meant acute coronary syndrome (ACS) and DM meant type 2 diabetes mellitus. ACS included STsegment elevated myocardial infarction (STEMI), non STsegment elevated myocardial infarction (NSTEMI) and unstable angina. ACS diagnosis was based on the clinical symptoms and signs, ischemic electrocardiographic abnormalities, and coronary angiography showing $\geq 50 \%$ stenosis in at least one main coronary artery. The exclusion criteria included: a history of renal failure, chronic hepatic diseases, high fever, or bacterial/viral infection, autoimmune disease, arthritis, malignancies, severe diabetes and hypertension, and other severe medical illnesses. Diagnosis of T2DM was based on one of the following criteria: fasting plasma glucose level of $\geq 126 \mathrm{mg} /$ $\mathrm{dL}(\geq 7.0 \mathrm{mmol} / \mathrm{L})$, random plasma glucose of $\geq 200 \mathrm{mg} /$ $\mathrm{dL}(\geq 11.1 \mathrm{mmol} / \mathrm{L})$, or plasma glucose of $\geq 200 \mathrm{mg} / \mathrm{dL}$ $(\geq 11.1 \mathrm{mmol} / \mathrm{L}$ ) after administration of $75 \mathrm{~g}$ oral glucose tolerance test (OGTT). The inclusion criteria were (i) being diagnosed with T2DM for more than 1 year, (ii) no antibiotics or steroidal and nonsteroidal anti-inflammatory medications being used during the last 3 weeks, and (iii) not treated with immunosuppressive chemotherapy, no current acute illness present, no professional periodontal treatment received during the last 6 months, and no ongoing pregnancy or lactation. Informed consent was obtained from each patient.

\section{Clinical and biochemical measurements}

Patient information, including age, gender, smoking and drinking history, and statin therapy history, was recorded. The details of anthropometric measurements (weight, height, body mass index) were assessed after overnight fasting for at least $10 \mathrm{~h}$. Peripheral blood samples were obtained from patients' brachial veins. Subjects fasted for at least $10 \mathrm{~h}$ before blood collection and then blood routine, urine routine, concentrations of lipid parameters, including total cholesterol (TC), triglyceride (TG), lowdensity lipoprotein cholesterol (LDL-C), HDL-C, apoAI, apoB, free fatty acid (FFA), were evaluated via standard laboratory procedures. Concentrations of high-sensitivity C-reactive protein (hsCRP) were measured with a latex particle, enhanced immunoturbidimetric assay. For the subsequent experiments, fresh plasma was obtained by centrifugation at $3000 \mathrm{r} / \mathrm{min}$ at $4{ }^{\circ} \mathrm{C}$ for $10 \mathrm{~min}$. The plasma was aliquoted and stored at $-80^{\circ} \mathrm{C}$ freezer until analysis.

\section{Measurement of plasma ANGPTL8}

Plasma ANGPTL8 concentration was measured with commercially available ELISA kits (ANGPTL8: EIAAB, E11644H, Wuhan, China). All the measurement of plasma ANGPTL8 was performed in duplicate for each sample. The coefficient of variation for intra- and interassay variation was $<6 \%$ and $<9 \%$, respectively.

\section{ApoB-depleted plasma preparation}

According to previous reported procedures [19], $540 \mu \mathrm{L}$ heparin sodium solution ( $280 \mathrm{mg} / \mathrm{mL}$, Aladdin, H104201) and $10 \mathrm{~mL}$ manganese chloride solution $(1.06 \mathrm{~mol} / \mathrm{L}$, Aladdin, M112542) were mixed. $100 \mu \mathrm{L}$ mixed solution was added to $1 \mathrm{~mL}$ plasma, incubated for $30 \mathrm{~min}$ at $4{ }^{\circ} \mathrm{C}$, followed by centrifugation at $1500 \mathrm{~g}$ for $30 \mathrm{~min}$. The supernatant was collected. If supernatant was still turbid (especially samples from patients with hypertriglyceridemia), plasma was centrifuged at $12000 \mathrm{~g}$ for $10 \mathrm{~min}$ again. The previous study revealed that heparin sodium/ manganese chloride precipitation had no effects on HDL size as well as cholesterol efflux measurement [20], and therefore this method was chosen to prepare apoBdepleted plasma in the study.

\section{Cholesterol efflux capacity measurement}

Cholesterol efflux experiments were performed according to established procedures [21, 22]. THP-1 human monocytes (ATCC) were grown in RPMI1640 medium (Gibco, 22400089), supplemented with $10 \%$ 
heat-inactivated FBS, 1\% penicillin/streptomycin until differentiation into macrophages by the addition of phorbol myristate acetate $(100 \mathrm{ng} / \mathrm{mL}$, Sigma, P1585). Subsequently, differentiated THP-1 macrophages were loaded with $50 \mu \mathrm{g} / \mathrm{mL}$ acetylated LDL (Peking UnionBiology Co. Ltd) and $1 \mu \mathrm{Ci} / \mathrm{mL}$ [3H] cholesterol for $24 \mathrm{~h}$. Macrophages were then washed twice with PBS (Gibco, 10010023) and equilibrated for $24 \mathrm{~h}$ in RPMI1640 medium containing $2 \%$ bovine serum albumin. Cells were then washed with PBS again and apoB-depleted plasma from individual patients was diluted in medium $(2.5 \%, \mathrm{vol} / \mathrm{vol})$. After $16 \mathrm{~h}$, the supernatant was collected and centrifuged to remove cellular debris. The cells were washed twice with PBS, and then incubated for at least $30 \mathrm{~min}$ at room temperature with $0.1 \mathrm{~mol} / \mathrm{L} \mathrm{NaOH}$ solution. The radioactivity within the supernatant and cells was determined by liquid scintillation counting. Wells incubated with RPMI1640 but without added apoBdepleted plasma were used as blanks, and these values were subtracted from the respective experimental values. Efflux is given as the percentage of counts recovered from the medium in relation to the total counts present on the plate (sum of medium and cells). All efflux experiments were performed in duplicate for each sample. The coefficient of variation for intra- and inter-assay variation was $<4 \%$ and $<8 \%$, respectively.

\section{Statistical analysis}

Statistical analysis was performed with Statistical Package for Social Sciences version 22.0 and plots were made with GraphPad Prism V.6.0 (GraphPad Software, Inc,
La Jolla, California, USA). Clinical data are expressed as mean \pm standard deviation (normally distributed continuous data) or median with interquartile range (skewed distributed continuous data). Comparisons between categorical data were performed with Chi-Squared tests, while continuous variables were assessed by unpaired $\mathrm{t}$ test (for normal distribution) or nonparametric test (for skewed distribution). To evaluate the associations between variables, Pearson correlation analysis was used. Stepwise multiple linear regression analysis was performed to determine the variables with independent significant association with cholesterol efflux capacity. These variables included all potential ones that might have a significant relationship with cholesterol efflux capacity in univariate analyses. In the correlation and regression analysis, logarithmic-transformed values were used for the variables skewed distributed. A two-tailed P value $<0.05$ was considered statistically significant.

\section{Results}

\section{Characteristics of subjects}

Demographic and biochemical characteristics of participants were shown in Table 1 . The study included 210 unrelated individuals, $60.9 \%$ of the participants were male and the mean age was 64.76 years. Compared to nonCAD controls, CAD subjects had significantly higher free fatty acid (FFA) and statin use, while other biomarkers are significantly lower, including total cholesterol (TC), high-density lipoprotein cholesterol (HDL-C), low-density lipoprotein cholesterol (LDL-C) and apolipoprotein AI (apoAI). When dividing the subjects into DM

Table 1 Baseline characteristics of all the subjects

\begin{tabular}{|c|c|c|c|c|c|c|}
\hline Variables & $\operatorname{CAD}(n=120)$ & nonCAD $(n=90)$ & $\mathbf{P}$ & $\mathrm{DM}(\mathrm{n}=37)$ & nonDM $(n=173)$ & $\mathbf{P}$ \\
\hline Male (\%) & 63.6 & 57.3 & NS & 59.8 & 61.3 & NS \\
\hline Age (years) & $63.96 \pm 7.85$ & $63.09 \pm 8.25$ & NS & $64.32 \pm 7.36$ & $63.41 \pm 8.18$ & NS \\
\hline BMI $\left(\mathrm{kg} / \mathrm{m}^{2}\right)$ & $24.17 \pm 3.92$ & $24.42 \pm 3.02$ & NS & $25.47 \pm 3.40$ & $24.00 \pm 3.54$ & 0.024 \\
\hline TG (mg/dl) & $128.43 \pm 71.74$ & $129.32 \pm 79.72$ & NS & $147.92 \pm 91.23$ & $124.00 \pm 69.97$ & NS \\
\hline $\mathrm{TC}(\mathrm{mg} / \mathrm{dl})$ & $145.40 \pm 35.96$ & $158.16 \pm 39.83$ & 0.005 & $146.95 \pm 40.22$ & $151.97 \pm 37.51$ & NS \\
\hline $\mathrm{HDL}-\mathrm{C}(\mathrm{mg} / \mathrm{dl})$ & $40.60 \pm 10.05$ & $44.08 \pm 11.21$ & 0.028 & $39.06 \pm 9.28$ & $42.92 \pm 10.83$ & NS \\
\hline LDL-C (mg/dl) & $88.16 \pm 30.16$ & $97.06 \pm 31.32$ & 0.021 & $88.17 \pm 31.32$ & $92.81 \pm 30.94$ & NS \\
\hline apoAl (g/L) & $1.08 \pm 0.20$ & $1.17 \pm 0.25$ & 0.008 & $1.08 \pm 0.20$ & $1.12 \pm 0.23$ & NS \\
\hline apoB (g/L) & $0.85 \pm 0.25$ & $0.89 \pm 0.26$ & NS & $0.89 \pm 0.28$ & $0.86 \pm 0.25$ & NS \\
\hline hsCRP (mg/L) & $4.93(1.26-13.44)$ & $2.36(1.17-7.00)$ & NS & $5.12(1.62-8.28)$ & $3.23(1.18-11.51)$ & NS \\
\hline FFA (mmol/L) & $0.52 \pm 0.29$ & $0.44 \pm 0.27$ & 0.047 & $0.57 \pm 0.29$ & $0.46 \pm 0.28$ & 0.045 \\
\hline Statin (\%) & 55.5 & 3.8 & $<0.0001$ & 35.1 & 32.9 & NS \\
\hline Smoking (\%) & 46.4 & 30.5 & 0.036 & 37.8 & 40.0 & NS \\
\hline HF (\%) & 72.7 & 42.7 & $<0.0001$ & 72.9 & 57.2 & NS \\
\hline
\end{tabular}

Values are expressed as mean \pm SD or median (interquartile range). CAD coronary artery disease, $D M$ diabetes mellitus, $B M I$ body mass index, $T G$ triglyceride, $T C$ total cholesterol, $H D L-C$ high-density lipoprotein cholesterol, $L D L-C$ low-density lipoprotein cholesterol, apoAl apolipoprotein $\mathrm{Al}$, apoB apolipoprotein $\mathrm{B}$, $h \mathrm{p} C R P$ high sensitivity $C$ reactive protein, $F F A$ free fatty acid, $H F$ heart failure 
group and nonDM group, DM subjects had significantly higher body mass index (BMI) and FFA level.

\section{ANGPTL8 concentration in subjects}

ANGPTL8 concentration between CAD and nonCAD groups was not significantly different [504.5 (364.4774.4) vs 452.8 (354.8-800.1), $\mathrm{P}=0.736]$. However, ANGPTL8 level in those with DM was significantly higher than those without DM [568.3 (406.2-836.8) vs 458.2 (356.8-755.6), $\mathrm{P}=0.023$, Fig. 1]. ANGPTL8 concentration in male subjects was also significantly higher than that in female subjects [535.8 (398.8-836.9) vs 430.3 (338.0-692.9), $\mathrm{P}=0.007]$. In addition, when subjects were divided into low triglyceride group (defined as $<150 \mathrm{mg} /$ $\mathrm{dL}$ ) and high triglyceride group (defined as $\geq 150 \mathrm{mg} / \mathrm{dL}$ ) according to previous report [23, 24], ANGPTL8 presented significantly higher level in low triglyceride group than in high triglyceride group [519.9 (377.6-809.0) vs 438.7 (329.2-681.0), $\mathrm{P}=0.038$, Fig. 2]. However, no significant difference of ANGPTL8 concentration was found between statin group and non-statin group [463.2 (356.6-745.3) vs 515.3 (377.4-831.3), $\mathrm{P}=0.139]$.

\section{Cholesterol efflux capacity in subjects}

Cholesterol efflux capacity in CAD subjects and DM subjects was significantly lower compared to nonCAD subjects and nonDM subjects (CAD: $12.51 \pm 2.83$ vs $14.58 \pm 2.06 \%, \mathrm{P}<0.0001$, Fig. 3 ; $\mathrm{DM}: 12.34 \pm 3.16$ vs $13.62 \pm 2.57 \%, P=0.0099$, Fig. 4).

\section{Correlation analysis of clinical variables in subjects}

To investigate variables associated with ANGPTL8 and cholesterol efflux capacity, correlation analysis was performed. In all the participants, plasma ANGPTL8 (log-transformed) presented a significant inverse relationship with cholesterol efflux capacity $(\mathrm{r}=-0.188, \mathrm{P}<0.01, \mathrm{n}=210$, Fig. 5). Plasma

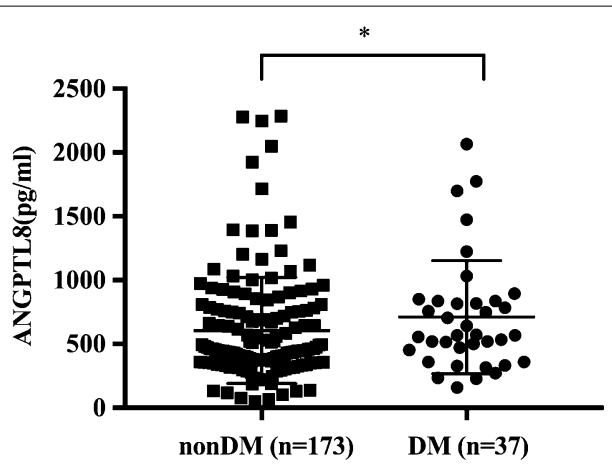

Fig. 1 ANGPTL8 from nonDM $(n=173)$ and DM $(n=37)$ patients. Data are expressed as median \pm interquartile range. DM indicates diabetes mellitus. ${ }^{*} \mathrm{P}<0.05$
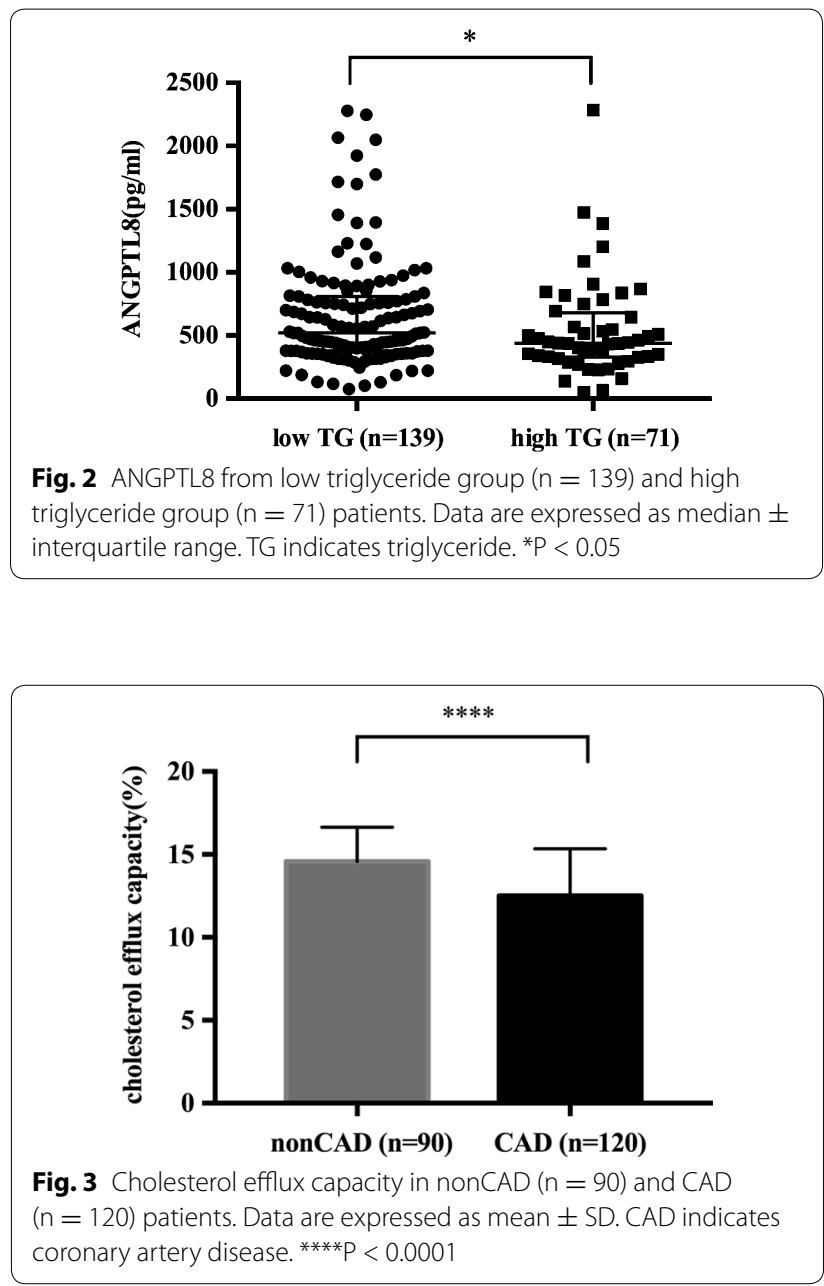

ANGPTL8 (log-transformed) is also inversely correlated with HDL-mediated cholesterol efflux capacity in CAD group $(\mathrm{r}=-0.247, \mathrm{P}<0.01, \mathrm{n}=120$, Additional file 1: Fig. S1) and nonDM group $(\mathrm{r}=-0.164, \mathrm{P}<0.05$, $\mathrm{n}=173$, Additional file 2: Fig. S2). At the same time, plasma ANGPTL8 (log-transformed) and cholesterol efflux capacity showed a significant correlation with other biomarkers (see Table 2).

\section{Multivariate analysis for the associations of clinical variables to cholesterol efflux capacity}

In order to determine the independent contributors to the cholesterol efflux capacity, stepwise multiple regression models were fitted after adjustment for different variables. Log-transformed values were used for the variables skewed distributed, including ANGPTL8 and hsCRP. The regression analysis revealed that ANGPTL8, apoAI and statin use were independent risk factors to the cholesterol efflux capacity (Table 3 ). 


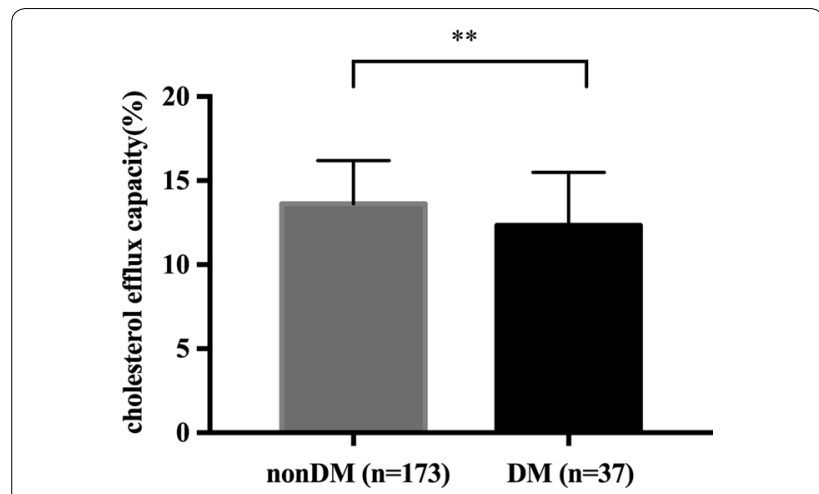

Fig. 4 Cholesterol efflux capacity in nonDM $(n=173)$ and DM $(n=37)$ patients. DM indicates diabetes mellitus. Data are expressed as mean $\pm S D$. ${ }^{* *} P<0.01$

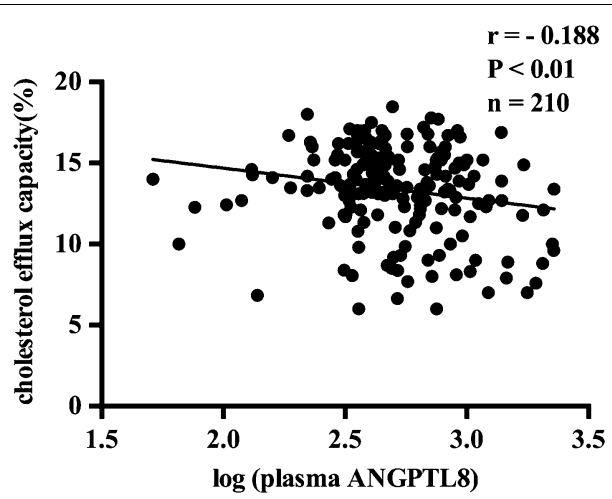

Fig. 5 The correlation between plasma ANGPTL8 (log-transformed) and cholesterol efflux capacity in all the subjects

Table 2 Pearson's correlations between clinical variables and log-transformed ANGPTL8 as well as cholesterol efflux capacity in all the subjects

\begin{tabular}{llllll}
\hline & \multicolumn{2}{l}{$\begin{array}{l}\text { ANGPTL8 } \\
\text { transformed) }\end{array}$} & & \multicolumn{2}{l}{$\begin{array}{l}\text { Cholesterol efflux } \\
\text { capacity }\end{array}$} \\
\cline { 2 - 3 } \cline { 5 - 6 } & $\mathbf{r}$ & $\mathbf{P}$ & & $\mathbf{r}$ & $\mathbf{P}$ \\
\hline Age & 0.250 & $<0.001$ & & -0.021 & $\mathrm{NS}$ \\
HDL-C & -0.075 & $\mathrm{NS}$ & & 0.402 & $<0.0001$ \\
LDL-C & -0.154 & 0.034 & & 0.216 & $<0.01$ \\
TC & -0.184 & 0.011 & & 0.266 & $<0.0001$ \\
TG & -0.099 & $\mathrm{NS}$ & & 0.130 & $\mathrm{NS}$ \\
ApoAl & -0.124 & $\mathrm{NS}$ & & 0.527 & $<0.0001$ \\
BUN & 0.347 & $<0.0001$ & -0.125 & $\mathrm{NS}$ \\
UA & 0.374 & $<0.0001$ & -0.251 & $<0.001$ \\
CR & 0.412 & $<0.0001$ & -0.126 & $\mathrm{NS}$ \\
hsCRP & 0.098 & $\mathrm{NS}$ & -0.244 & $<0.001$ \\
(Log-transformed) & & & & \\
\hline
\end{tabular}

ANGPTL8 angiopoietin-like protein 8, HDL-C high density lipoprotein-cholesterol, $L D L-C$ low density lipoprotein-cholesterol, $T C$ total cholesterol, TG triglyceride, apoAl apolipoprotein $\mathrm{Al}, \mathrm{BUN}$ blood urea nitrogen, $U A$ uric acid, $C R$ creatinine, hs $C R P$ high-sensitivity $C$ reactive protein

\section{Discussion}

In this study, we found that ANGPTL8 showed no significant difference between CAD and nonCAD groups, while ANGPTL8 in DM group was significantly higher compared to nonDM controls. Besides, ANGPTL8 was inversely correlated with HDL-mediated cholesterol efflux capacity. Stepwise multiple regression analysis revealed that ANGPTL8 was an independent contributor to HDL-mediated cholesterol efflux capacity.

\section{ANGPTL8 and blood lipids}

ANGPTL8 has been reported to present significant but contradictive relationship with various blood lipid markers. Genomic studies showed that ANGPTL8 gene variant was associated with $\mathrm{HDL}-\mathrm{C}$ variant $[16,25]$. The relationship between ANGPTL8 and triglyceride has also been deeply excavated. A recently published paper reported that ANGPTL8 knockout mediated by CRISPR-Cas9 system led to reduced plasma triglyceride levels [26], which verified conclusions from animal experiments-ANGPTL8 was found to inhibit LPL activity and elevate plasma triglyceride partly via ANGPTL3 activation after feeding [14]. A prospective cohort study conducted in Korean children revealed that baseline ANGPTL8 was associated with future changes in triglyceride levels [27]. However, in our research, ANGPTL8 presented no correlation with triglyceride and HDL-C level, even though ANGPTL8 was inversely associated with LDL-C and total cholesterol. Results from epidemiological studies are inconsistent $[16-18,24]$. The relationship between ANGPTL8 and blood lipid markers might be confounded by sample selection and age, which is positively correlated with ANGPTL8 in our study and other literature [28]. Besides, a study focused on the effects of vitamin D on the relationship between ANGPTL8 and cardiometabolic risk factors has found that the association varied in different vitamin D status [23]. In our study, patients were not stratified by vitamin D status, and it might be a potential reason for the discrepancies among studies.

Of note, ANGPTL8 presented a strongly positive relationship with biomarkers of renal function in our study, including BUN, UA and CR. A previous research studied the association of ANGPTL8 and diabetic nephropathy and found that ANGPTL8 was predictive of the progression of diabetic nephropathy [29]. Abnormal ANGPTL8 elevation may lead to dysregulated lipid metabolism in the kidney, which offers a possible explanation for the role of ANGPTL8 in the development of diabetic nephropathy. 
Table 3 Stepwise multiple regression analysis detecting independent contributors to HDL-mediated cholesterol efflux capacity in all the subjects

\begin{tabular}{lccr}
\hline Factors & $\boldsymbol{\beta}$ & Standardized $\boldsymbol{\beta}$ & \multicolumn{1}{l}{$\mathbf{P}$} \\
\hline apoAI & 0.057 & 0.481 & $<0.001$ \\
ANGPTL8 & -0.014 & -0.143 & 0.023 \\
(log-transformed) & & & \\
hsCRP & -0.002 & -0.048 & 0.482 \\
(log-transformed) & & & \\
LDL-C & 0.001 & 0.028 & 0.675 \\
Statin use & -0.008 & -0.140 & 0.027 \\
\hline
\end{tabular}

R square: $0.316, P<0.001$

\section{ANGPTL8 and HDL function}

HDL-mediated cholesterol efflux capacity was inversely associated with cardiovascular risk independent of HDL-C concentration [7, 9, 30]. The ability of HDL to mediate cholesterol efflux from lipid-laden peripheral macrophages is the crucial part of HDL atheroprotective function. In inflammatory conditions, such as coronary artery disease, the lipid and protein composition of HDL can also be altered, a process which is called HDL remodeling. HDL inflammatory remodeling could impair cholesterol efflux. Our previous study found that apoAI modification mediated by myeloperoxidase oxidation could impair cholesterol efflux capacity [22]. Increased serum amyloid A(SAA) in HDL particles could result in HDL trapping in the extracellular matrix and impair HDL-mediate cholesterol efflux [31,32].

Our previous study revealed that apolipoprotein CIII(apoCIII) enrichment was inversely correlated with HDL-mediated cholesterol efflux [33]. However, the relationship between apoCIII and HDL-mediated cholesterol efflux was independent of inflammatory biomarker such as hsCRP level, therefore, we hypothesized that apoCIII might play an atherogenic role independent of its pro-inflammatory effects. In this study, we found that ANGPTL8 concentration was an independent contributor to cholesterol efflux capacity after controlling for hsCRP. A previous study showed that ANGPTL8 was increased in metabolic syndrome patients and presented a strongly positive correlation with hsCRP [28], implying a potential role of ANGPTL8 in inflammation. However, Zhang et al. [34] found that ANGPTL8 functioned as a negative regulator in nuclear factor- $\kappa B$ activation triggered by tumor necrosis factor $\alpha$. Therefore, it seems invalid that ANGPTL8 may impair HDL-mediated cholesterol efflux via inflammation-related pathways.

ApoAI removes cholesterol from peripheral cells via membrane transporter ATP-binding cassette
A1(ABCA1) to form nascent HDL particles [35]. Increased unsaturated fatty acids can enhance $A B C A 1$ degradation and reduce membrane ABCA1 content, leading to impaired cholesterol efflux mediated by ABCA1 pathway [36]. Dang et al. [37] found that ANGPTL8 overexpression could dramatically elevate non-esterified fatty acids content. Based on these findings, we hypothesized that ANGPTL8 might impair HDL-mediated cholesterol efflux via the positive effects on unsaturated fatty acids and subsequent ABCA1 decrease.

ANGPTL8 could promote ANGPTL3 cleavage and form a complex with the cleaved $\mathrm{N}$-terminal of ANGPTL3 [14]. The complex was demonstrated to inhibit LPL activity and modulate lipid metabolism. The close relationship between ANGPTL3 and ANGPTL8 casts a shadow over the studies which are designed to explore the independent effects of ANGPTL3 or ANGPTL8 on HDL function. Zhao et al. [38] studied the association between ANGPTL3 and HDL-mediated cholesterol efflux on T2DM subjects. Their findings showed that plasma ANGPTL3 increase was positively associated with apoAI content in HDL particles from female diabetic patients, however, plasma ANGPTL3 increase presented a weak and insignificant inverse relationship with change of HDL-mediated cholesterol efflux [38]. It is highly possible that ANGPTL3 and ANGPTL8 could reside on HDL particles and those ANGPTL3 and ANGPTL8 molecules residing on HDL particles were the real contributors to HDL functional change.

Although HDL-mediated cholesterol efflux was thought to play an important role in atheroprotection and found to be inversely associated with atherosclerosis incidence, however, a previous study reported that increased cholesterol efflux to apoB-depleted serum was positively associated with incident cardiovascular events [39]. The paradoxical findings implicated the limitations of cholesterol efflux assay. Excessive cholesterol needs to be removed from peripheral macrophages to apoAI or HDL and return to the liver for biliary excretion. In vitro cholesterol efflux assay cannot fully demonstrate the whole reverse cholesterol transport pathway and the validity should be carefully interpreted.

\section{Conclusions}

Our study presented that ANGPTL8 exerted a negative effect on HDL-mediated cholesterol efflux capacity, which provided evidence for the role of ANGPTL8 in HDL dysfunction and a possible explanation for the atherogenic effects of ANGPTL8. However, further research is warranted to elucidate the underlying mechanism of ANGPTL8 effects. 


\section{Additional files}

Additional file 1: Figure S1. The correlation between plasma ANGPTL8 (log-transformed) and cholesterol efflux capacity in CAD subjects.

Additional file 2: Figure S2. The correlation between plasma ANGPTL8 (log-transformed) and cholesterol efflux capacity in nonDM subjects.

\section{Abbreviations}

CAD: coronary artery disease; DM: diabetes mellitus; ANGPTL8: angiopoietinlike protein 8; HDL: high density lipoprotein; LDL: low density lipoprotein; TG: triglyceride; TC: total cholesterol; BMI: body mass index; BUN: blood urea nitrogen; UA: uric acid; CR: creatinine; hsCRP: high-sensitivity $C$ reactive protein; apoAl: apolipoprotein Al; apoB: apolipoprotein B; FFA: free fatty acid.

\section{Authors' contributions}

$M L$ and DP designed the study and performed the data analysis. ML and ZZ performed the experiments. ML, YP and SW prepared the manuscript. All authors read and approved the final manuscript.

\section{Author details}

${ }^{1}$ Department of Cardiovascular Medicine, The Second Xiangya Hospital, Central South University, No.139, Middle Renmin Road, Changsha 410011, Hunan, China. ${ }^{2}$ Department of Metabolism \& Endocrinology, The Second Xiangya Hospital, Central South University, Changsha, Hunan, China.

\section{Acknowledgements}

Not applicable.

\section{Competing interests}

The authors declare that they have no competing interests.

\section{Availability of data and materials}

All data generated or analysed during the study are available from the corresponding author on reasonable request and included in this published article.

\section{Consent for publication}

All participants provided written informed consent before enrollment in this study.

\section{Ethics approval and consent to participate}

The study protocol conforms to the ethical guidelines of the 1975 Declaration of Helsinki as reflected in a priori approval by the Medical Ethics Committee of the Second Xiangya Hospital of Central South University.

\section{Funding}

This project was supported by grants from National Natural Science Foundation of China (No.81670426 to D.P.).

\section{Publisher's Note}

Springer Nature remains neutral with regard to jurisdictional claims in published maps and institutional affiliations.

Received: 9 July 2018 Accepted: 31 October 2018

Published online: 08 November 2018

\section{References}

1. Emerging Risk Factors C, Di Angelantonio E, Sarwar N, Perry P, Kaptoge S, Ray KK, Thompson A, Wood AM, Lewington S, Sattar N, et al. Major lipids, apolipoproteins, and risk of vascular disease. JAMA. 2009;302(18):1993-2000.

2. Boekholdt SM, Arsenault BJ, Hovingh GK, Mora S, Pedersen TR, Larosa JC, Welch KM, Amarenco P, Demicco DA, Tonkin AM, et al. Levels and changes of $\mathrm{HDL}$ cholesterol and apolipoprotein A-I in relation to risk of cardiovascular events among statin-treated patients: a meta-analysis. Circulation. 2013;128(14):1504-12.
3. Investigators A-H, Boden WE, Probstfield JL, Anderson T, Chaitman BR, Desvignes-Nickens P, Koprowicz K, McBride R, Teo K, Weintraub W. Niacin in patients with low HDL cholesterol levels receiving intensive statin therapy. N Engl J Med. 2011;365(24):2255-67.

4. Keene D, Price C, Shun-Shin MJ, Francis DP. Effect on cardiovascular risk of high density lipoprotein targeted drug treatments niacin, fibrates, and CETP inhibitors: meta-analysis of randomised controlled trials including 117,411 patients. BMJ. 2014;349:g4379.

5. Jansen H, Samani NJ, Schunkert H. Mendelian randomization studies in coronary artery disease. Eur Heart J. 2014;35(29):1917-24.

6. Rosenson RS, Brewer HB Jr, Davidson WS, Fayad ZA, Fuster V, Goldstein J, Hellerstein M, Jiang XC, Phillips MC, Rader DJ, et al. Cholesterol efflux and atheroprotection: advancing the concept of reverse cholesterol transport. Circulation. 2012;125(15):1905-19.

7. Khera AV, Cuchel M, de la Llera-Moya M, Rodrigues A, Burke MF, Jafri K, French BC, Phillips JA, Mucksavage ML, Wilensky RL, et al. Cholesterol efflux capacity, high-density lipoprotein function, and atherosclerosis. N Engl J Med. 2011;364(2):127-35.

8. Wu S, Chen X, Yuan S, Peng D, Zhou A, Li J. Impaired cholesterol efflux capacity is related to increased carotid intima media thickness in patients with end-stage renal disease. Int J Cardiol. 2015;187:456-8.

9. Rohatgi A, Khera A, Berry JD, Givens EG, Ayers CR, Wedin KE, Neeland IJ, Yuhanna IS, Rader DR, de Lemos JA, et al. HDL cholesterol efflux capacity and incident cardiovascular events. N Engl J Med. 2014;371(25):2383-93.

10. Ronsein GE, Vaisar T. Inflammation, remodeling, and other factors affecting HDL cholesterol efflux. Curr Opin Lipidol. 2017;28(1):52-9.

11. Dijk W, Kersten S. Regulation of lipid metabolism by angiopoietin-like proteins. Curr Opin Lipidol. 2016;27(3):249-56.

12. Zhang R, Abou-Samra AB. A dual role of lipasin (betatrophin) in lipid metabolism and glucose homeostasis: consensus and controversy. Cardiovasc Diabetol. 2014;13:133.

13. Fu Z, Abou-Samra AB, Zhang R. A lipasin/Angptl8 monoclonal antibody lowers mouse serum triglycerides involving increased postprandial activity of the cardiac lipoprotein lipase. Sci Rep. 2015;5:18502.

14. Quagliarini F, Wang Y, Kozlitina J, Grishin NV, Hyde R, Boerwinkle E, Valenzuela DM, Murphy AJ, Cohen JC, Hobbs HH. Atypical angiopoietin-like protein that regulates ANGPTL3. Proc Natl Acad Sci U S A. 2012;109(48):19751-6.

15. Kersten S. Physiological regulation of lipoprotein lipase. Biochim Biophys Acta. 2014;1841(7):919-33.

16. Hanson RL, Leti F, Tsinajinnie D, Kobes S, Puppala S, Curran JE, Almasy L, Lehman DM, Blangero J, Duggirala $R$, et al. The Arg59Trp variant in ANGPTL8 (betatrophin) is associated with total and HDL-cholesterol in American Indians and Mexican Americans and differentially affects cleavage of ANGPTL3. Mol Genet Metab. 2016;118(2):128-37.

17. Morinaga J, Zhao J, Endo M, Kadomatsu T, Miyata K, Sugizaki T, Okadome Y, Tian Z, Horiguchi H, Miyashita K, et al. Association of circulating ANGPTL 3, 4, and 8 levels with medical status in a population undergoing routine medical checkups: a cross-sectional study. PLOS ONE. 2018;13(3):e0193731.

18. Pascual-Corrales E, Gomez-Ambrosi J, Moncada R, Valenti V, Catalan V, Rodriguez A, Ramirez B, Silva C, Gil MJ, Salvador J, et al. Circulating ANGPTL8/betatrophin concentrations are increased after surgically induced weight loss, but not after diet-induced weight loss. Obes Surg. 2016;26(8):1881-9.

19. Warnick GR, Albers JJ. A comprehensive evaluation of the heparin-manganese precipitation procedure for estimating high density lipoprotein cholesterol. J Lipid Res. 1978;19(1):65-76.

20. Davidson WS, Heink A, Sexmith H, Melchior JT, Gordon SM, Kuklenyik Z, Woollett L, Barr JR, Jones JI, Toth CA, et al. The effects of apolipoprotein $B$ depletion on HDL subspecies composition and function. J Lipid Res. 2016;57(4):674-86.

21. Triolo M, Annema W, de Boer JF, Tietge UJ, Dullaart RP. Simvastatin and bezafibrate increase cholesterol efflux in men with type 2 diabetes. Eur J Clin Invest. 2014;44(3):240-8.

22. Peng DQ, Brubaker G, Wu Z, Zheng L, Willard B, Kinter M, Hazen SL, Smith $J D$. Apolipoprotein A-I tryptophan substitution leads to resistance to myeloperoxidase-mediated loss of function. Arterioscler Thromb Vasc Biol. 2008;28(11):2063-70.

23. Fu J, Hou C, Li L, Feng D, Li G, Li M, Li C, Gao S, Li M. Vitamin D modifies the associations between circulating betatrophin and cardiometabolic 
risk factors among youths at risk for metabolic syndrome. Cardiovasc Diabetol. 2016;15(1):142.

24. Gomez-Ambrosi J, Pascual-Corrales E, Catalan V, Rodriguez A, Ramirez B, Romero S, Vila N, Ibanez P, Margall MA, Silva C, et al. Altered concentrations in Dyslipidemia evidence a role for ANGPTL8/betatrophin in lipid metabolism in humans. J Clin Endocrinol Metab. 2016;101(10):3803-11.

25. Cannon ME, Duan Q, Wu Y, Zeynalzadeh M, Xu Z, Kangas AJ, Soininen P, Ala-Korpela M, Civelek M, Lusis AJ, et al. Trans-ancestry fine mapping and molecular assays identify regulatory variants at the ANGPTL8 HDL-C GWAS Locus. G3 (Bethesda). 2017;7(9):3217-27.

26. Izumi R, Kusakabe T, Noguchi M, Iwakura H, Tanaka T, Miyazawa T, Aotani D, Hosoda K, Kangawa K, Nakao K. CRISPR/Cas9-mediated Angptl8 knockout suppresses plasma triglyceride concentrations and adiposity in rats. J Lipid Res. 2018;59(9):1575-85.

27. Chung HS, Lee MJ, Hwang SY, Lee HJ, Yoo HJ, Seo JA, Kim SG, Kim NH, Baik SH, Choi DS, et al. Circulating angiopoietin-like protein 8 (ANGPTL8) and ANGPTL3 concentrations in relation to anthropometric and metabolic profiles in Korean children: a prospective cohort study. Cardiovasc Diabetol. 2016;15:1.

28. Abu-Farha M, Abubaker J, Al-Khairi I, Cherian P, Noronha F, Kavalakatt S, Khadir A, Behbehani K, Alarouj M, Bennakhi A, et al. Circulating angiopoietin-like protein 8 (betatrophin) association with $\mathrm{HsCRP}$ and metabolic syndrome. Cardiovasc Diabetol. 2016;15:25.

29. Chen CC, Susanto H, Chuang WH, Liu TY, Wang CH. Higher serum betatrophin level in type 2 diabetes subjects is associated with urinary albumin excretion and renal function. Cardiovasc Diabetol. 2016;15:3.

30. Qiu C, Zhao X, Zhou Q, Zhang Z. High-density lipoprotein cholesterol efflux capacity is inversely associated with cardiovascular risk: a systematic review and meta-analysis. Lipids Health Dis. 2017;16(1):212.
31. Vaisar T, Tang C, Babenko I, Hutchins P, Wimberger J, Suffredini AF, Heinecke JW. Inflammatory remodeling of the HDL proteome impairs cholesterol efflux capacity. J Lipid Res. 2015;56(8):1519-30.

32. Han CY, Tang C, Guevara ME, Wei H, Wietecha T, Shao B, Subramanian S, Omer M, Wang S, O'Brien KD, et al. Serum amyloid A impairs the antiinflammatory properties of HDL. J Clin Invest. 2016;126(2):796.

33. Luo M, Liu A, Wang S, Wang T, Hu D, Wu S, Peng D. ApoClll enrichment in HDL impairs HDL-mediated cholesterol efflux capacity. Sci Rep. 2017;7(1):2312.

34. Zhang Y, Guo X, Yan W, Chen Y, Ke M, Cheng C, Zhu X, Xue W, Zhou Q, Zheng $L$, et al. ANGPTL8 negatively regulates NF-kappaB activation by facilitating selective autophagic degradation of IKKgamma. Nat Commun. 2017:8(1):2164.

35. Brownell N, Rohatgi A. Modulating cholesterol efflux capacity to improve cardiovascular disease. Curr Opin Lipidol. 2016;27(4):398-407.

36. Wang Y, Oram JF. Unsaturated fatty acids inhibit cholesterol efflux from macrophages by increasing degradation of ATP-binding cassette transporter A1. J Biol Chem. 2002;277(7):5692-7.

37. Dang F, Wu R, Wang P, Wu Y, Azam MS, Xu Q, Chen Y, Liu Y. Fasting and feeding signals control the oscillatory expression of Angptl8 to modulate lipid metabolism. Sci Rep. 2016;6:36926.

38. Zhao D, Yang LY, Wang XH, Yuan SS, Yu CG, Wang ZW, Lang JN, Feng YM. Different relationship between ANGPTL3 and HDL components in female non-diabetic subjects and type-2 diabetic patients. Cardiovasc Diabetol. 2016;15(1):132.

39. Li XM, Tang WH, Mosior MK, Huang Y, Wu Y, Matter W, Gao V, Schmitt D, Didonato JA, Fisher EA, et al. Paradoxical association of enhanced cholesterol efflux with increased incident cardiovascular risks. Arterioscler Thromb Vasc Biol. 2013;33(7):1696-705.
Ready to submit your research? Choose BMC and benefit from:

- fast, convenient online submission

- thorough peer review by experienced researchers in your field

- rapid publication on acceptance

- support for research data, including large and complex data types

- gold Open Access which fosters wider collaboration and increased citations

- maximum visibility for your research: over 100M website views per year

At BMC, research is always in progress.

Learn more biomedcentral.com/submissions 